\title{
Cancer
}

\section{Functions of ras oncogenes}

\section{from Chris Marshall}

ONE of the fundamental tenets of cancer research is that the conversion of normal cells to tumour cells is a multistage process. A possible mechanism would involve two or more genetic alterations, perhaps affecting different cellular functions. Studies with mesenchymal and epithelial cells in culture have provided some clues as to how this might work. They have identified two distinct differences between normal cells and tumour cells. The first is that tumour cells generally have the capacity to divide forever - they are immortalized. And second, their growth is controlled abnormally. Thus tumour cells, unlike normal cells, will often proliferate in low levels of serum mitogens or in the absence of anchorage. Frequently associated with abnormal growth control are changes in cell morphology. The existence of immortalized cell lines such as 3T3, which show normal growth control and are non-tumorigenic, and the demonstration for two tumour viruses, polyoma and adenovirus, that the functions of immortalization and abnormal growth control are genetically separate ${ }^{1-3}$, suggest that different genes may be involved in conferring these two phenotypes on tumour cells.

The question then is, which genes in tumour cells are involved in immortalization and which confer morphological transformation and abnormal growth control? A year ago three papers published in Nature led to the same conclusion: activated ras oncogenes, detected by their ability to transform the established fibroblast cell line NIH 3T3, were not capable of immortalizing non-established cells but could morphologically transform and confer abnormal growth control on immortalized cells and, under certain conditions, non-immortalized cells ${ }^{4-6}$. A paper appearing in this issue of Nature comes to a different conclusion: under appropriate conditions, transfection with ras genes can lead to immortalization. These results clearly differ from those published previously, but they are still consistent with multistage models of neoplastic transformation because to produce the tumorigenic phenotype, both the expression and coding potential of the ras gene had to be altered.

Spandidos and Wilkie ${ }^{7}$ have transfected non-established strains of Chinese hamster and rat cells with mutant and normal ras genes either under the control of their own promoters or in plasmids that also contain transcriptional enhancers from simian virus $\mathbf{4 0}$ or the Moloney murine leukaemia virus long terminal repeat. They find that cells transfected with an activated c-Haras- 1 oncogene cloned from the T24 bladder carcinoma cell line are immortalized but not morphologically transformed or anchorage-independent. When cells were transfected with the oncogene under the control of a transcriptional enhancer they were immortalized, morphologically transformed, anchorage-independent and tumorigenic. Further, while the normal c-Ha-ras-1 gene alone did not affect the cells, when coupled to a transcriptional enhancer it immortalized non-tumorigenic cells.

The reasons for the discrepancy between the earlier experiments and those of Spandidos and Wilkie are not entirely clear. Experiments using the same constructs but in Syrian hamster cells have not resulted in immortalization (R.F. Newbold, personal communication). The experiments of Spandidos and Wilkie, however, point to the importance of achieving the appropriate degree of expression of transfecting genes, and this degree may be subtly different in different cell types.

Another possible explanation for the different results is suggested by the observation that cells differ in their response to exogenously supplied transforming growth factors (TGFs) and that this reflects the ease with which they can be transformed ${ }^{8}$. Thus, differences in response to TGFs by different cell types may explain why, in some cases, a ras oncogene can morphologically transform non-established cell $s^{4,5}$, while in Spandidos and Wilkie's experiments the T24 oncogene on its own did not. Interestingly, immortalization seems to be associated with an increase in sensitivity to $\mathrm{TGFs}^{8}$. Differences in responsiveness to TGFs and the degree of expression of a transfecting gene may also explain the intriguing observation that human fibroblasts are resistant to the effect of a transfecting ras oncogene ${ }^{9}$. Human fibroblasts are also resistant to transformation by Kirsten murine sarcoma virus (KiMSV) ${ }^{10}$.

What is not clear at this stage is whether experiments with ras-containing retroviruses really help to resolve the issue of whether ras immortalizes cells. Although innoculation of Harvey, Kirsten or BALB murine sarcoma viruses produces tumours, immortalization may not be a requirement for tumour formation by sarcoma virus because the virus stocks always contain a non-transforming helper virus, and a tumour can grow both by cell division and by recruitment from spreading viral infection. It would be of great interest to know whether the cells in these tumours have the ability to proliferate endlessly in culture. Infection of non-established rat embryo cells in culture with KiMSV only leads to morphological transformation at very low efficiency and such cells do not become immortalized (ref.8 and B.W. Ozanne, personal communication).

Nevertheless, it looks as though ras genes, like the myc gene ${ }^{4}$, may function as immortalization genes. It is important to note, however, that none of the experiments reported so far has demonstrated that the immortalized phenotype of the transfected cells is directly under the control of the transfecting gene. This has been done so far only in the case of the large $T$ gene of polyoma virus. Using a temperature-sensitive mutant it has now been possible to show that a functional large-T protein is necessary to maintain the immortalized phenotype ${ }^{11}$. Such experiments are critical because of the propensity of rodent cells to undergo spontaneous neoplastic transformation. The rates of neoplastic transformation differ between species, for example, the frequency of establishment of cell lines from Chinese hamster is about 10 times higher than that of Syrian hamster cells ${ }^{12}$.

Introduction of an oncogene into cells is only one way to analyse transformation. On page 508 of this issue, another approach is described. Stacey and Kung have micro-injected into various cell types purified p21 ras protein from BALB murine sarcoma virus expressed in Escherichia coli $^{13}$. Unsurprisingly, though comfortingly, micro-injection into NIH 3 T 3 cells results in morphological transformation. No transformation occurs when the protein is injected into nonestablished human diploid cells, a result which is reminiscent of those of Sager ${ }^{9}$ and Kopelovitch ${ }^{10}$. Of more novelty is the finding that when non-dividing NIH 3T3 cells are micro-injected with BALB MSV p21, the cells start to synthesize DNA. Such an effect is independent of serum growth factors since when quiescent NIH $3 \mathrm{~T} 3$ or NRK cells are micro-injected in serum-free media with p21 ras derived from the T24 c-Ha-ras oncogenes, they undergo rapid proliferation (James J. Feramisco, personal communication). These results, coupled with the observations that ras expression varies through the cell cycle ${ }^{14}$ and that the $\mathrm{p} 21$ ras proteins interact with the receptor for epidermal growth factor ${ }^{15}$, suggest that one function of the normal ras gene product may be in transducing signals from growth factor receptors. Mutant ras genes may be capable of giving the signal in the presence of levels of growth factors which are insufficient to make normal cells divide. Whether immortalization depends on such a function of the $\mathrm{p} 21$ ras protein remains to be determined.

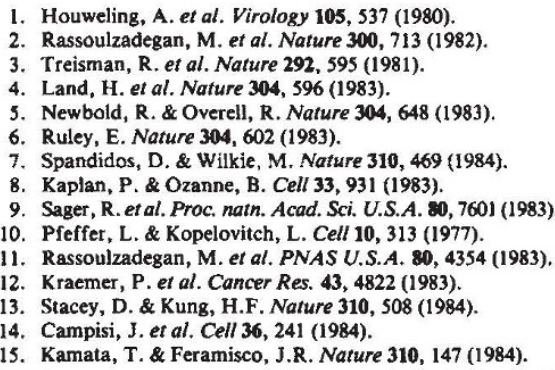

